Classification

Physics Abstracts

$07.80-78.70-71.45 \mathrm{G}$

\title{
Interpretation of valence loss spectra from composite media
}

\author{
Archie Howie and Caroline Walsh \\ Cavendish Laboratory, Madingley Road, Cambridge CB3 OHE, England \\ (Received October 31, 1990; accepted January 28, 1991)
}

\begin{abstract}
We discuss the interpretation of valence EELS data from composite media containing defects, clusters or precipates which may be difficult to resolve individually because of their small size or because of overlap effects. In some cases a well-defined collective loss (plasmon) peak is observed the position of which shifts slightly with changing defect concentration. In other cases discussed here the most noticeable effect is the appearance of new peaks in the spectrum which can be identified with interface as well as bulk excitations. Effective medium theories, although widely used to interpret optical absorption data from such samples, are only partially successful in explaining the energy loss spectra observed and do not account for the bulk loss peak from the minority phase. A simple dielectric excitation model where an appropriate average is made over the trajectories of a classical particle in the composite medium appears to offer a much better explanation and could be a useful way of characterising the fine-scale structure of composite samples. The connection between this trajectory approach and effective medium theory is discussed.
\end{abstract}

\section{Introduction.}

Because of the relatively high intensities available, valence loss spectroscopy emerged as one of the most successful techniques when transmission electron microscopists first began to address problems of microanalysis. Photographic and effectively parallel recording systems were employed allowing the spectra to be combined with image information in one $[1,2]$ or two $[3,4]$ dimensions. The considerable number of interesting and useful results which were obtained, particularly in free electron alloys, has been comprehensively reviewed [5].

With the advent of sector magnet systems attached to the scanning transmission electron microscope (STEM), interest for microanalysis largely transferred to the relatively weaker but much more directly interpretable core loss part of the energy loss spectrum. Activity in developing the theory of valence loss spectroscopy for inhomogeneous media has nevertheless continued and the applicability of the dielectric excitation theory for a fast classical charged particle has been established for the STEM situation [6]. The response of each local region is assumed to be described by an appropriate frequency-dependent, complex dielectric function $\varepsilon(\omega)$. Solutions have been worked out for a number of cases of practical interest including planar interfaces $[7,8]$, spheres [9-11] and cylinders [12]. Experimental results show that the dielectric excitation theory is capable of predicting the loss spectra in quantitative detail, allowing for instance fully consistent dielectric characterisation of an interface or small particle [13]. In some cases the spectra contain details 
relating to significant microstructural features which cannot be resolved in the usual electron microscope image. Thus Walls [14] (see also [13]) suggested the presence of an $\sim 1 \mathrm{~nm}$ layer of SiO at the $\mathrm{Si}-\mathrm{SiO}_{2}$ interface on the basis of an analysis of the observed intensity of the $8 \mathrm{eV}$ interface plasmon. Ugarte [15] studied Si spheres with an oxide coating but found in the loss spectrum an unexpected peak at 3-4 eV localised at the external surface. He ascribed the presence of this peak to a $0.5 \mathrm{~nm}$ thick layer of $\mathrm{Si}$ formed after reduction of the external oxide surface.

Another example recently studied where valence loss spectroscopy gives information about specimen structure on the near atomic scale is the case of dealuminated zeolites [16]. With increasing dealumination, the main collective loss peak observed at about $23 \mathrm{eV}$ moves systematically downwards by about $1 \mathrm{eV}$. Although zeolites are large band-gap insulators, this behaviour is reminiscent of that observed many years ago [5] for free electron alloys as the valence electron density is changed. A systematic analysis of the zeolite data [16], led to a determination of the change in valence electron density during dealumination and to some deductions about the possible nature of the defect sites created.

There are other examples of fine-scale two phase structures which yield a valence loss spectrum exhibiting behaviour which is considerably more complex than that observed for zeolites or free electron alloys. In addition to shifts in the main loss peak as a function of composition, one may observe the appearance of fresh peaks in the loss spectrum some of which can be attributed to the generation of excitations at the interfaces between the phases. Here we present results for the case of small $\mathrm{Al}$ particles in a matrix of $\mathrm{AlF}_{3}$ and discuss them in terms of dielectric excitation theory for a two phase medium. Development of a reliable theory for the interpretation of such data could lay a foundation for the application of electron energy loss spectroscopy to a new range of challenging and important microstructural problems.

\section{Experimental observations.}

It is well known that thanks to the extremely high current density in the focussed spot produced in the STEM, fine holes can be drilled in thin samples of many oxides and other non-conducting inorganic solids. In the case of $\mathrm{AlF}_{3}$ which has been studied by a number of workers, valence loss spectra have been obtained from inside individual holes as well as from the neighbouring region [12]. As a result of slight damage, the region immediately surrounding the hole drilled by a stationary beam has sometimes been transformed into a structure of small colloidal Al particles embedded in $\mathrm{AlF}_{3}$. A similar colloidal structure can also be produced over a large area by scanning the beam. Such an area is shown in figure 1, imaged in various signals obtained in the STEM. Detailed interpretation of these images is complicated by the great amount of overlapping detail as well as by thickness changes. The annular dark field (ADF) signal shown in figure 1a is generated by electrons which have been scattered through relatively large angles from individual atoms in the beam. In this instance the contrast probably arises from loss of mainly $F$ atoms as a result of beam damage combined possibly with pre-existing local variations in the specimen thickness. Thus the small dark area arrowed is a partially drilled hole formed where the beam pauses before each fresh scan of the larger rectangular area. The presence of the small $\mathrm{Al}$ particles is far from clear in the $\mathrm{ADF}$ image, probably because the scattered intensity from them is estimated to be only about $15 \%$ greater than that from a similar volume of $\mathrm{AlF}_{3}$. The small particles are more clearly visible in figures $1 \mathrm{~b}$ and $\mathrm{c}$ which are taken respectively with electrons of $6 \mathrm{eV}$ and $15 \mathrm{eV}$ energy loss corresponding to the two prominent new peaks which, as shown in figure 2, appear in the loss spectrum after damage. From the behaviour of these peaks as a function of damage and hence increasing $\mathrm{Al}$ content, they have been tentatively identified [12] with the interface and bulk excitation associated with the small $\mathrm{Al}$ particles. It should also be noted that, when the beam is 
passed through a hole already drilled and surrounded by a colloidal region [12], the spectrum still exhibits the interface loss peak but the bulk $\mathrm{Al}$ loss at $15 \mathrm{eV}$ is no longer visible.

(a)

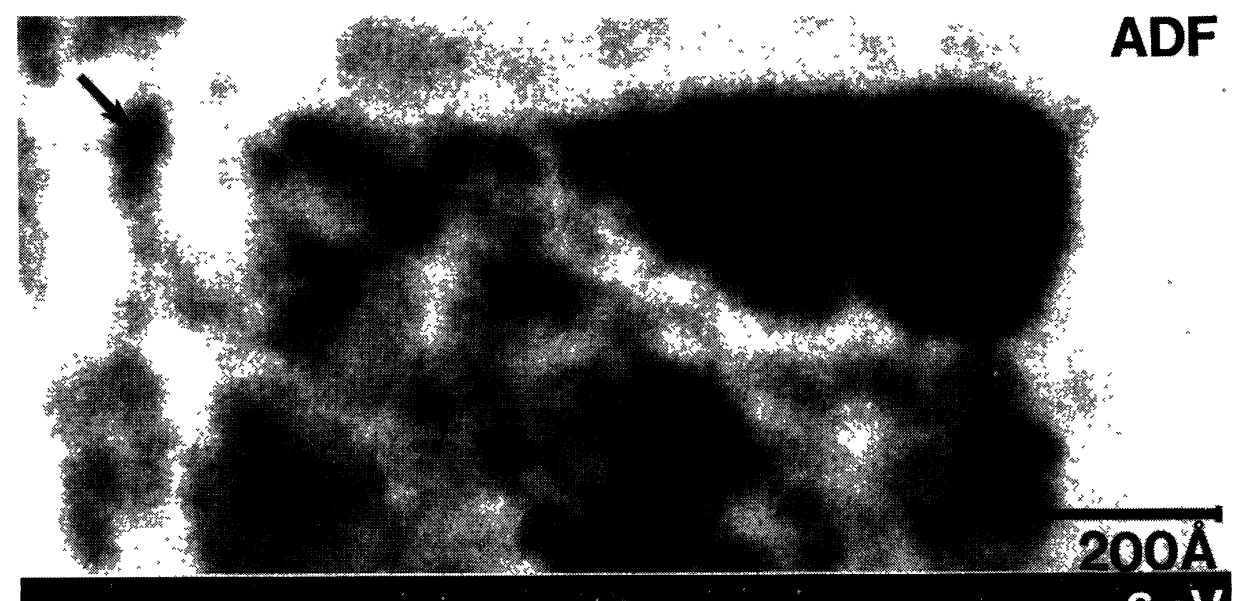

(b)

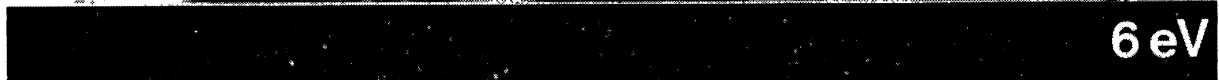

(c)

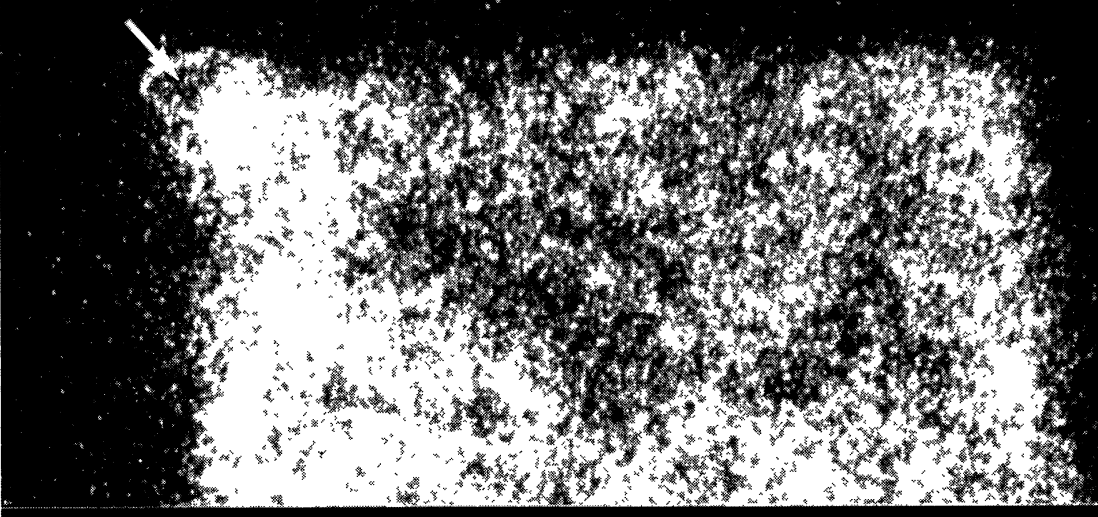

$15 \mathrm{eV}$

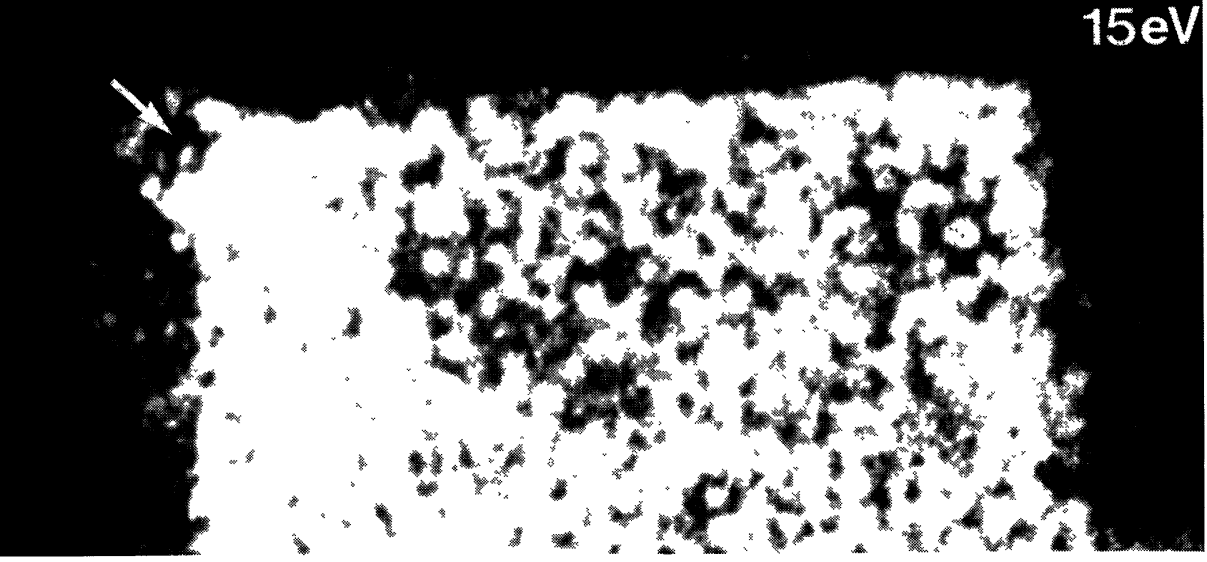

Fig. 1. - STEM images of a slightly damaged region produced in $\mathrm{AlF}_{3}$ by scanning a rectangular area. The arrow indicates a hole partially drilled where the beam paused at the start of each scan. The annular dark field image is shown in (a), while (b) and (c) are taken with $6 \mathrm{eV}$ and $15 \mathrm{eV}$ energy loss electrons respectively. 


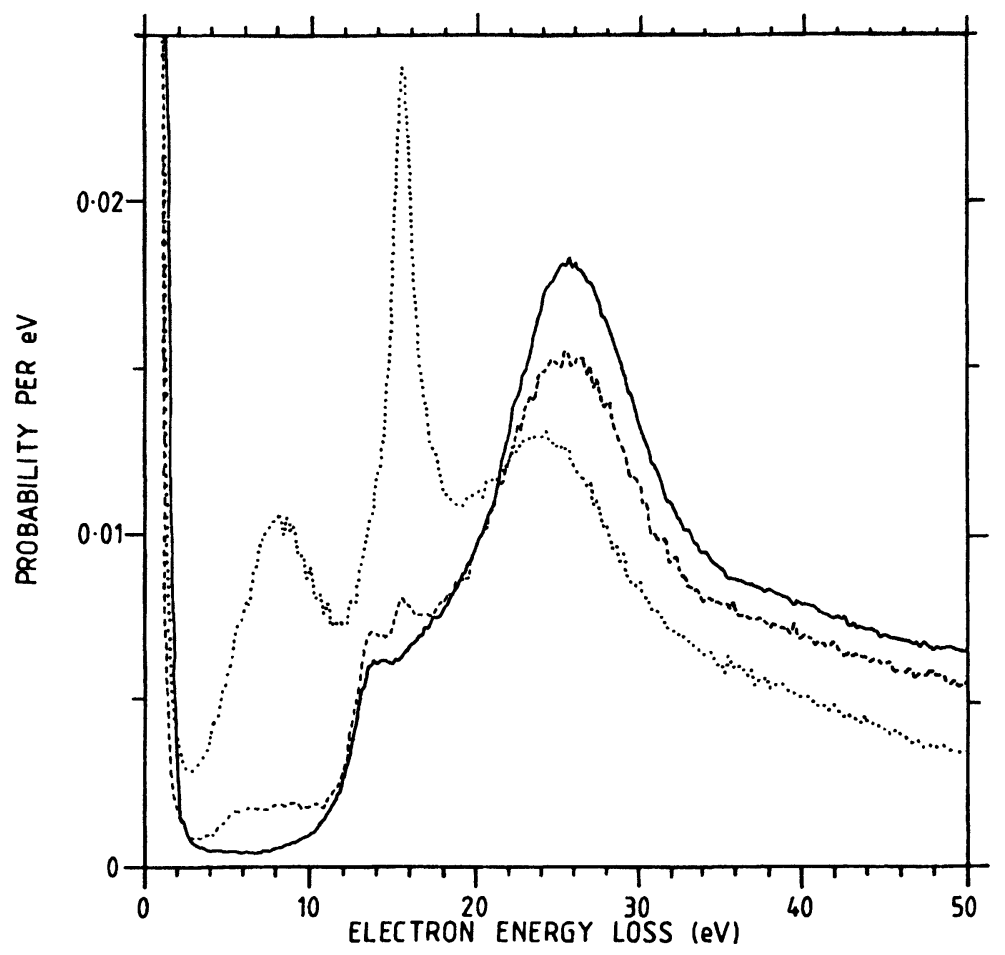

Fig. 2. - Energy loss spectra taken from $\mathrm{AlF}_{3}$ before appreciable damage and after increasing amounts of damage leading to new losses at $6-8 \mathrm{eV}$ and $16 \mathrm{eV}$.

\section{Effective dielectric response theory.}

An obvious approach to theoretical modelling of the experimental results just described is via effective medium theory whereby the behaviour of the composite medium is described by an effective dielectric response function $\varepsilon_{\text {eff }}$. There are a large number of recipes for $\varepsilon_{\text {eff }}$ but we consider first three of the simplest ones applicable to a medium consisting of a volume fraction $f$ of small particles of material A embedded in a second material B.

3.1 SimPLE AVERAGING. - Simple averaging of the dielectric response functions yields the result

$$
\varepsilon_{\mathrm{eff}}=\bar{\varepsilon}=f \varepsilon_{\mathrm{A}}+(1-f) \varepsilon_{\mathrm{B}}
$$

In free electron materials, where $\varepsilon(\omega)=1-\left(n e^{2} / \varepsilon_{0} \mathrm{~m}\right) / \omega(\omega+i \gamma)$, this procedure clearly corresponds to averaging of the valence electron density $n$ and would thus supply an explanation (though perhaps not the only explanation) of the results already obtained [5] on these materials. The plasmon loss in $\mathrm{Al}+\mathrm{Mg}$ alloys of varying composition for instance appears to follow the change in average electron density at least to a first approximation.

3.2 MAXWELL-GARNETT EFFECTIVE MEDIUM THEORY. - Maxwell-Garnett effective medium theory [17], which is a generalisation of the well known Clausius-Mosotti theory for the static 
dielectric constant, uses the expression

$$
\varepsilon_{\mathrm{eff}}=\varepsilon_{\mathrm{B}} \frac{\left((1+2 f) \varepsilon_{\mathrm{A}}+2(1-f) \varepsilon_{\mathrm{B}}\right)}{\left((1-f) \varepsilon_{\mathrm{A}}+(2+f) \varepsilon_{\mathrm{B}}\right)}
$$

This theory is restricted to small particles of material A and takes some account (in the dipole approximation) of the inter-particle interactions which become important at larger values of $f$. It agrees reasonably well with a considerable number of optical studies, particularly of small conducting particles in an insulating medium when a specific absorption peak is found, often identified with the vanishing of the denominator on the right hand side of equation (2). The MaxwellGarnett theory fails however to exhibit the percolation threshold which occurs at higher values of $f$ in such systems and treats the two media A and B in a curiously unsymmetrical way.

3.3 BRUGGEMAN EFFECTIVE MEDIUM THEORY. - The Bruggeman effective medium theory [18] was derived for a composite medium consisting of two materials both in the form of small spheres. It depends on the equation

$$
f \frac{\varepsilon_{\mathrm{eff}}-\varepsilon_{\mathrm{A}}}{2 \varepsilon_{\mathrm{eff}}+\varepsilon_{\mathrm{A}}}+(1-f) \frac{\varepsilon_{\mathrm{eff}}-\varepsilon_{\mathrm{B}}}{2 \varepsilon_{\mathrm{eff}}+\varepsilon_{\mathrm{B}}}=0
$$

At very small values of $f$ it can readily be shown that the Bruggeman and Maxwell-Garnett theories are equivalent. The two materials are clearly treated in a symmetrical way in equation (3) and a percolation transition is in fact predicted. The Bruggemann theory is however not generally superior to the Maxwell-Garnett theory in describing optical properties at higher values of $f$.

3.4 MiXTURE OF CLOSELY SIMILAR DIELECTRICS. - For the case when two closely similar dielectrics are mixed together on a fine scale, Landau and Lifshitz [19] derive a general result equivalent to

$$
\varepsilon_{\text {eff }}=\bar{\varepsilon}-f(f-1)\left(\varepsilon_{\mathrm{A}}-\varepsilon_{\mathrm{B}}\right)^{2} / 3 \bar{\varepsilon}
$$

Although the systems of interest here do not fall within the scope of this theory, it is interesting to note that equations (2) and (3) are both, in the appropriate limit, consistent with equation (4). The simple averaging theory represented by equation (1) is however not consistent with equation (4).

\section{Analysis of $\mathrm{Al} / \mathrm{AlF}_{3}$ data.}

4.1 Application of EXISTING EFFeCtive MEdium theories. - The ability of the theories represented by equations (1), (2) and (3) above to describe the loss spectra obtained in the $\mathrm{Al} / \mathrm{AlF}_{3}$ system was investigated and the results are presented in figures $3 a$ and $b$ for two different values of the volume fraction $f$ of the Al phase A.

When phase $\mathrm{A}$ is present as larger clusters, which may as in figure 1 above even be visible in the electron microscope, new features including interface excitations and bulk excitations from phase A as well as phase B can be observed. Equation (1) does not in general predict additional features of this kind. One may expect a systematic shift of any collective loss peak with energy well above the band gap of either material. For an insulator containing a small volume fraction of metallic inclusions there can also be a new collective peak with energy well below the band gap corresponding to the vanishing of the right hand side of the equation. The physical significance of this peak whose position depends sharply on the value of $f$ is unclear however. It would not correspond to a maximum of $\operatorname{Im}\left(\varepsilon_{\text {eff }}\right)$ and hence cannot be associated with any peak in optical 


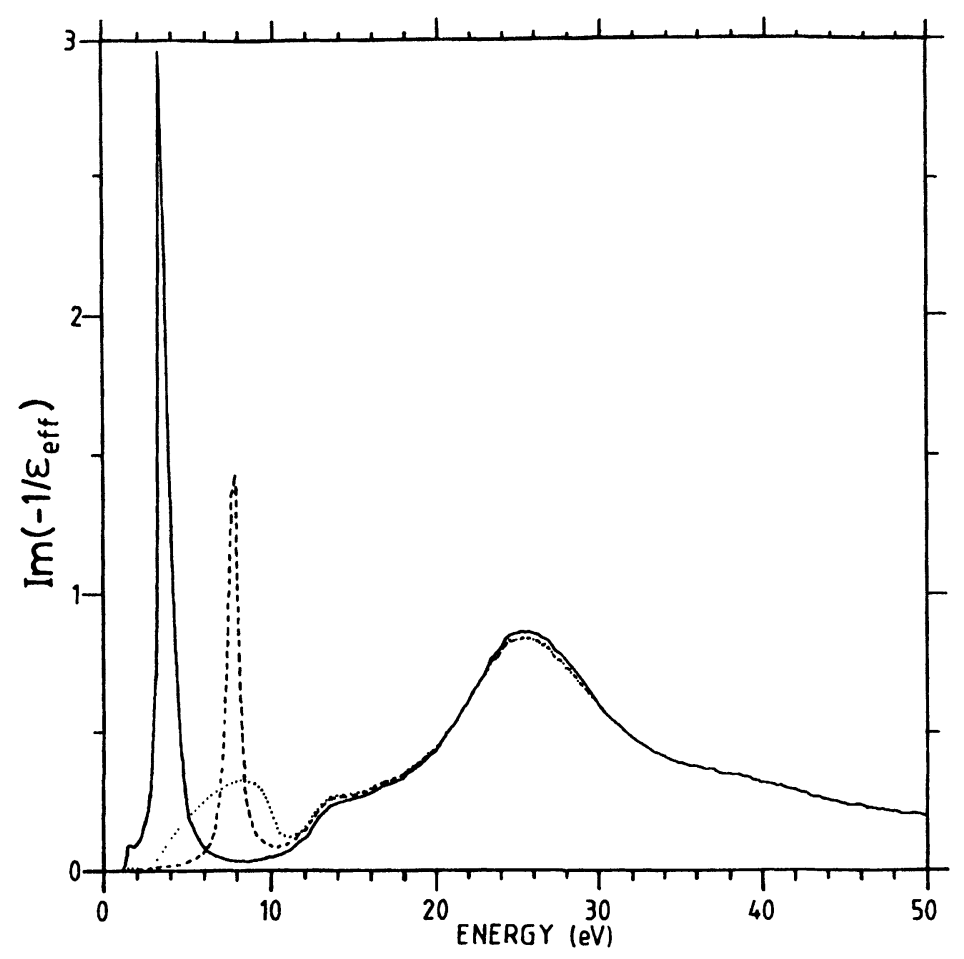

(a)

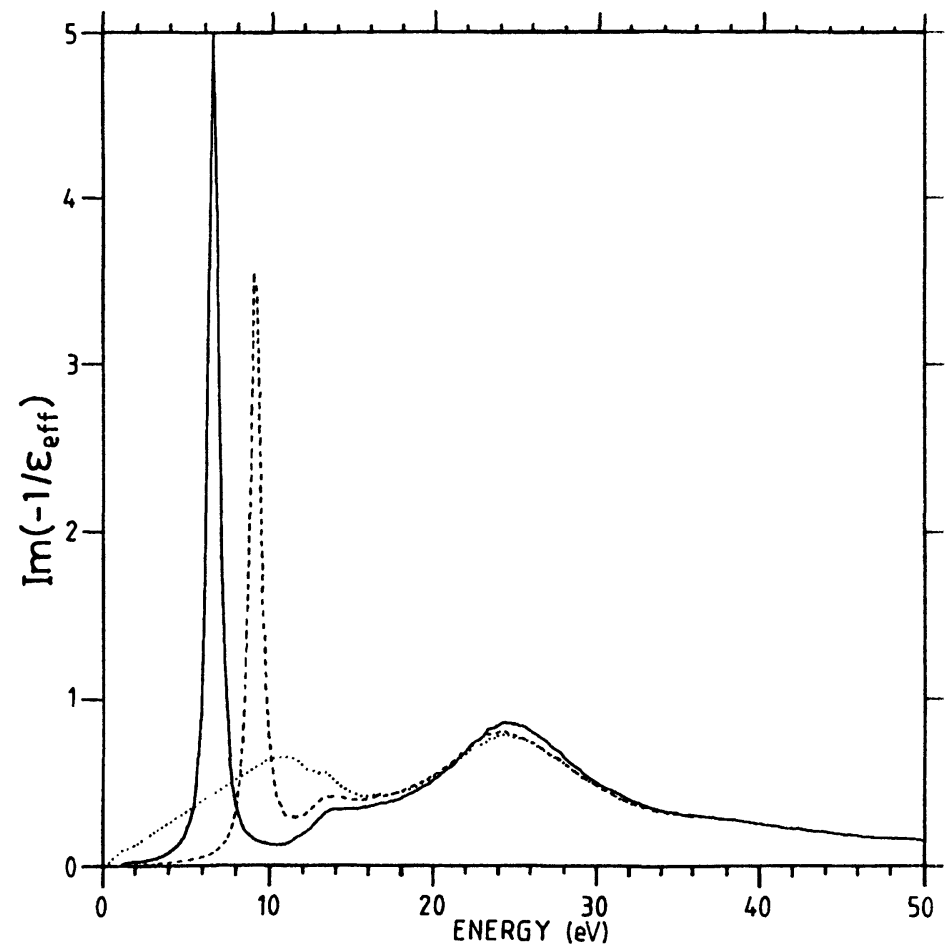

(b)

Fig. 3. - The effective energy loss function $\operatorname{Im}\left(-1 / \varepsilon_{\text {eff }}\right)$ calculated for the $\mathrm{Al} / \mathrm{AlF}{ }_{3}$ system on the simple averaging model (continuous curve), the Maxwell-Garnett theory (broken curve) and the Bruggeman theory (dotted curve). The curves in (a) and (b) are for $f=0.1$ and 0.3 respectively. 
absorption. Figure 3 shows loss spectra computed [12] using equations (1), (2) and (3) for $\mathrm{AlF}_{3}$ containing various volume fractions $f$ of $\mathrm{Al}$. The dielectric function $\varepsilon_{\mathrm{B}}$ used here was derived from experimental loss spectra obtained from undamaged $\mathrm{AlF}_{3}$. It is clear tha none of the three theories is capable of explaining the occurrence of the $15 \mathrm{eV}$ loss peak which is almost certainly associated with the excitation of bulk plasmons in the $\mathrm{Al}$ precipitates. The interface peak is less well accounted for by equation (1) but is moderately well explained by both the Maxwell Garnett and Bruggeman theories. It can be shown that the general equivalence of these two theories at small values of $f$ fails near a zero of $\varepsilon_{A}+2 \varepsilon_{B}$. As discussed by Walsh [20] the fact that neither theory accounts precisely for the position and width of the observed peak at $6 \mathrm{eV}$ may well be due to their neglect of relativistic effects as well of the finite particle size and the variation in particle size and shape. When more sophisticated effective medium theories $[21,22]$, designed to take better account of finite particle size and interaction effects are employed [20], there is still a complete failure to account for the presence of the Al bulk loss peak.

4.2 EXCITATION THEORY BASED ON ELECTRON TRAJECTORIES. - An early theory of dielectric excitations in small spheres was worked out by Fujimoto and Komaki [23] and predicts both bulk and surface excitations created by a parallel unfocussed electron beam. This approach involves integrating over impact parameters and is not immediately applicable to the situation in the STEM, which involves excitation by a focussed electron beam with a definite impact parameter relative to the sphere [24]. For trajectories outside the sphere theoretical analysis shows that interface modes for many values of the angular momentum $l$ can be generated $[9,10]$ as well as bulk modes in any surrounding dielectric medium. Internal trajectories can give rise to both bulk and surface excitations of the sphere $[11,24,25]$.

If we work directly with the two bulk loss functions $\operatorname{Im}\left(-1 / \varepsilon_{\mathrm{A}}\right)$ and $\operatorname{Im}\left(-1 / \varepsilon_{\mathrm{B}}\right)$ as well as the interface loss function $\operatorname{Im}\left\{-3 /\left(\varepsilon_{\mathrm{A}}+2 \varepsilon_{\mathrm{B}}\right)\right\}$ which is appropriate for a small sphere of material $\mathrm{A}$ embedded in material $\mathrm{B}$, we can easily write down an effective excitation function by averaging over the different parts of a typical trajectory,

$$
\begin{aligned}
& \operatorname{Im}\left(-\frac{1}{\varepsilon_{\text {eff }}}\right)=f\left(\operatorname{Im}\left(\frac{-1}{\varepsilon_{\mathrm{A}}}\right)+g_{\text {int }}\left(\operatorname{Im}\left(-\frac{3}{\varepsilon_{\mathrm{A}}+2 \varepsilon_{\mathrm{B}}}\right)-\operatorname{Im}\left(-\frac{1}{\varepsilon_{\mathrm{A}}}\right)\right)\right) \\
& +(1-f)\left(\operatorname{Im}\left(-\frac{1}{\varepsilon_{\mathrm{B}}}\right)+g_{\mathrm{ext}}\left(\operatorname{Im}\left(-\frac{3}{\varepsilon_{\mathrm{A}}+2 \varepsilon_{\mathrm{B}}}\right)-\operatorname{Im}\left(-\frac{1}{\varepsilon_{\mathrm{B}}}\right)\right)\right)
\end{aligned}
$$

In this expression the main terms with factors $f$ and $(1-f)$ describe the contributions from trajectory segments inside and outside the particles respectively. For each of these cases the inner terms with factors $g_{\text {int }}$ and $g_{\text {ext }}$ give the interface contribution as well as the corresponding reduction in the bulk contribution (the so-called Begrenzungs correction). The form of both these inner terms is consistent with the previous theoretical work on spheres $[10,11,23]$ in the limit of small sphere radius where only the dipole interface mode is excited.

The parameter $g_{\text {int }}$ in equation (5) determines the relative importance of interface to bulk excitations in the small spheres of material A. The dielectric excitation theory of planar interfaces indicates that interface excitations are generated from segments of the trajectory within a distance of about $v / \omega$ of the interface where $v$ is the velocity of the fast electron and $\hbar \omega$ is the energy of the excitation. We therefore expect $g_{\text {int }}=1$ for spheres of radius $a$ much less than $v / \omega$. For spheres of large radius we expect $g_{\text {int }}$ to be proportional to $v / a \omega$. A simple expression, consistent with these ideas and with the numerical results quoted by Echenique et al. [11], is proposed here.

$$
g_{\text {int }}=1 /(1+3 a \omega / v)
$$


It is interesting to note that the Maxwell-Garnett equation (2) can be rewritten in the form

$$
\operatorname{Im}\left(-\frac{1}{\varepsilon_{\text {eff }}}\right)=\operatorname{Im}\left(-\frac{1}{\varepsilon_{\mathrm{B}}}\right)+\frac{3 f}{(1+2 f)}\left(\operatorname{Im}\left(-\frac{3}{(1+2 f) \varepsilon_{\mathrm{A}}+2(1-f) \varepsilon_{\mathrm{B}}}\right)-\operatorname{Im}\left(-\frac{1}{\varepsilon_{\mathrm{B}}}\right)\right)
$$

For small spheres $\left(g_{\text {int }}=1\right)$ and small values of $f$, this equation is consistent not only with the work of Fujimoto and Komaki [23], in the appropriate limit, but also agrees exactly with the trajectory model of equation (5) provided we assume the relations

$$
\begin{aligned}
f+(1-f) g_{\text {ext }} & =3 f /(1+2 f) \\
\text { i.e. } \quad g_{\text {ext }} & =2 f /(1+2 f)
\end{aligned}
$$

The $g_{\text {ext }}$ factor effectively represents the volume fraction of material B, which is close enough to a piece of material A to give rise to an interface excitation. The above dependence of $g_{\text {ext }}$ on $f$ therefore seems quite reasonable, at least for small values of $f$. An alternative evaluation of $g_{\text {ext }}$ could presumably be made by averaging the expression [10] for the probability of interface excitation on a sphere over a range of impact parameters appropriate for a given value of $f$. It would also be of interest to compare equation (5) with a modified form of the Fujimoto and Komaki expression [23] by suitably restricting the integration over impact parameters.

At larger values of $f$, the position of the interface excitation predicted by equation (7) shifts, possibly as a result of interparticle interactions. As $f$ approaches 1 this interface term goes smoothly over to the bulk loss term for material A. Equation (7) also makes very clear however the result previously noted that the Maxwell-Garnett theory will not produce any bulk plasmon contribution from the spheres, for small or even moderate values of the volume fraction $f$.

Results obtained from the improved theory described by equations (5), (6) and (8) are shown in figure 4 for the $\mathrm{Al} / \mathrm{AlF}_{3}$ system using a number of values of the two parameters $f$ and $a$. It is clear that this two parameter model allows a better qualitative fit to the observations to be made, including not only the interface loss peak but also the bulk loss peaks in both the A and B materials. A more quantitative fit could probably only be obtained by using a considerably more sophisticated model. In practice the observed spectra are probably complicated by the presence of defect states and voids in addition to the $\mathrm{Al}$ particles, all created in the damage process.

\section{Discussion.}

With some further development, the trajectory averaging model presented here could form the basis of an extremely useful technique for the characterisation of specimens with microstructure which is too complex and fine scale to be directly imaged in the transmission electron microscope. Such specimens could include samples containing highly dispersed precipitate systems or high densities of point defect clusters. It might for example be possible to determine the pore volume and pore size in catalyst support materials more reliably than can be done by high resolution electron microscopy. The valence loss spectra required for such assessments could be obtained by transmission through thin samples as described here but could also in principle be generated by passing the fast electron beam parallel to and just outside a flat surface of a much thicker sample. It should be noted however, that for such external trajectories, equation (5) would have to be modified since the beam does not pass through either of the two media and the bulk loss excitations would be completely absent. This observation is entirely consistent with the results.

A number of improvements should probably be made to the theory however. In the denominator of the interface excitation in equation (5), the expression $\left(\varepsilon_{A}+2 \varepsilon_{B}\right)$ could perhaps be 


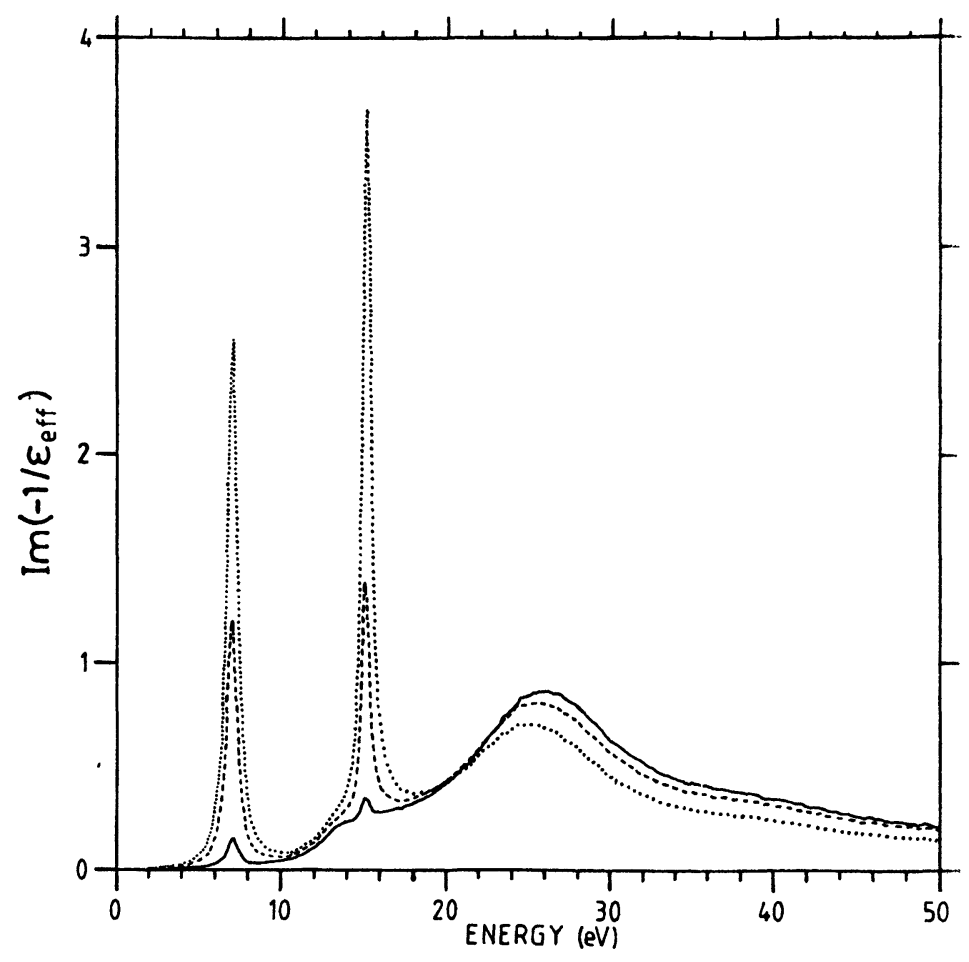

(a)

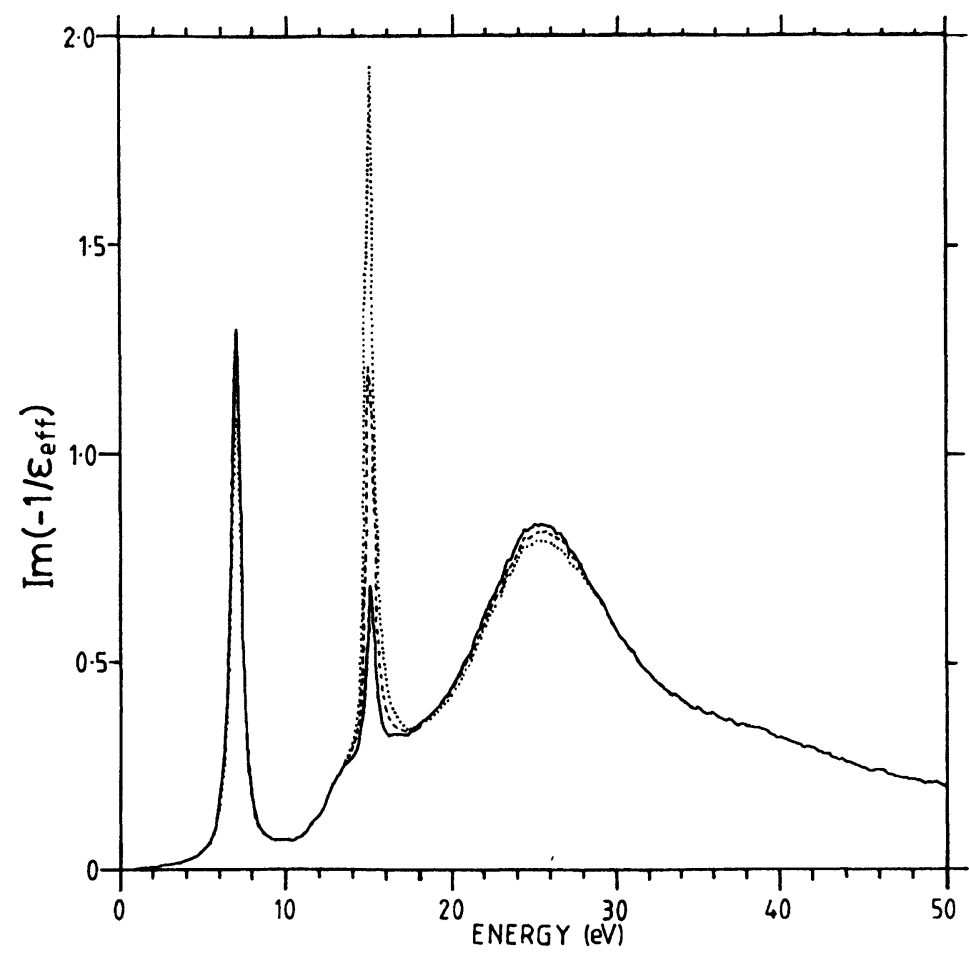

(b)

Fig. 4. - The effective energy loss function $\operatorname{Im}\left(-1 / \varepsilon_{\text {eff }}\right)$ calculated for the $\mathrm{Al} / \mathrm{AlF}_{3}$ system on the basis of equation (5). The curves in (a) are for $a=2 \mathrm{~nm}, f=0.01,0.1$ and 0.3 ; those in (b) are for $f=0.1$, $a=0.5 \mathrm{~nm}, a=1.5 \mathrm{~nm}$ and $a=5 \mathrm{~nm}$ and show the increase in magnitude of the bulk Al loss relative to the surface loss with increasing particle size. 
replaced by $\left([1+f] \varepsilon_{\mathrm{A}}+[2-f] \varepsilon_{\mathrm{B}}\right)$. This would then have the correct dipole limiting form for small spheres A in material B when $f$ is close to zero, going over to small spheres B in material A when $f$ is close to 1 . At intermediate values of $f$, the excitation function would then be closer to that appropriate for planar interfaces. More realistically of course, the interface excitation function should depend on the parameter $a$ which is used for the sphere radius or more generally to characterise the scale of the microstructure.

Interaction effects between neighbouring spheres or inclusions, which have been observed experimentally [25], may become important, particularly for intermediate values of $f$. These were not explicity included in the arguments used to produce equation (4) but the similarity with the Maxwell-Garnett theory for these interactions as represented by equation (5) suggests that it may be possible to include such effects in the theory with only minor modifications.

An obvious way to check and improve the theory given here would be to carry out systematic optical absorption and valence electron energy loss spectroscopy on a range of two-phase samples whose microstructure could be independently characterised in terms of the parameters $f$ and $a$ using transmission electron microscopy. The $\mathrm{Al} / \mathrm{AlF}_{3}$ system used here is somewhat unsuitable because of its tendency to damage during observation. Optical absorption, electron energy loss and electron microscopy studies have been carried out on the $\mathrm{Au} / \mathrm{SiO}_{2}$ system $[26,27]$ which shows a well-defined interface loss whose position seems to depend on both $a$ and $f$. Unfortunately however, the bulk loss peaks in both $\mathrm{Au}$ and $\mathrm{SiO}_{2}$ are not very sharp so that their presence is not very noticeable. We have confirmed that the $\mathrm{Au} / \mathrm{SiO}_{2}$ data can be fitted by equation (5). A more crucial test of equation (5) clearly requires a system which exhibits sharp and readily identifable interface and bulk losses for all the materials present. Equations (5) and (6) both show that the origin of peaks in the optical or energy loss spectrum of a composite medium can be more complicated than has been generally been thought. Thus an optical absorption peak, associated with a maximum in $\operatorname{Im}\left(\varepsilon_{\text {eff }}\right)$, could arise from (amongst other things) a zero of $\left\{(1-f) \varepsilon_{A}+(2+f) \varepsilon_{B}\right\}$, as indicated by equation (2), or from a zero of $\left\{(1+2 f) \varepsilon_{\mathrm{A}}+2(1-f) \varepsilon_{\mathrm{B}}\right\}$ as shown by equation (7). In the second case however, since the immediate consequence is a maximum in $\operatorname{Im}\left(-1 / \varepsilon_{\text {eff }}\right)$, the final result could be a collective loss rather than an absorption peak.

\section{Acknowledgements.}

$\mathrm{AH}$ is grateful to Gatan Inc for financial support enabling this paper to be presented in initial form at the Lake Tahoe EELS meeting. CAW thanks the SERC for a research assistantship.

\section{References}

[1] MOLlENSTEDT G., Optik 5 (1949) 499.

[2] METHERELl A.J.F. and WHELAN M.J., J. Appl. Phys. 237 (1966) 1737.

[3] CASTAing R. and Henry L., C.r. Hebd. Seanc. Acad. Sci (Paris) 255 (1962) 76.

[4] Castaing R., Henoc P., Henry L. and NatTa M., C.r. Hebd. Seanc. Acad. Sci (Paris) 265 (1967) 1293.

[5] Metherell A.J.F., Adv. in Opt. and El. Micr. V.E. Cosslett and R. Barer Eds. (Academic Press) 4 (1971) 263.

[6] Ritchie R.H. and Howie A., Philos. Mag. A58 (1988) 753.

[7] ECHENIQUe P.M. and PENDRY J.B., J. Phys. C8 (1975) 2936.

[8] Garcia Molina R., Gras Marti A., Howie A. and Ritchie R.H., J. Phys C18 (1985) 5335.

[9] FERrEll T.L. and ECHENIQUe P.M., Phys. Rev. Lett. 55 (1985) 1526. 
[10] EcheniQue P.M., Howie A. and Wheatley D.J., Philos. Mag. B56 (1987) 335.

[11] ECHENIQUe P.M., BAUSElis J. and Rivacoba A., Phys. Rev. B35 (1987) 1521.

[12] WALSH C.A., Phil. Mag. 59 (1989) 227.

[13] WALIS M.G. and HowIE A., Ultramicroscopy 28 (1989) 40.

[14] Walls M.G. Ph.D Thesis (Univ. of Cambridge) 1988.

[15] UgarTe D., Doctor of Science Thesis (Univ. of Paris-Sud) 1990.

[16 McCOMB D. and HowIE A., Ultramicroscopy (1990) in press.

[17] MaXWell GarnetT J.C., Phil. Trans. Roy. Soc. 203 (1904) 385.

[18] Bruggeman D.A.G., Ann. Phys. (Leipz.) 24 (1935) 636.

[19] LANDAU L.D. and LIFSHITZ E.M., Electrodynamics of Continuous Media (Pergamon) 1960.

[20] WALSH C.A., PhD Thesis (Univ. of Cambridge) 1989.

[21] STROUd D. and PAN F.P., Phys. Rev. B17 (1978) 1602.

[22] WACHNIEWSKI A. and McClung H.B., Phys. Rev. B33 (1986) 8053.

[23] Fujimoto F. and KomaKi K., J. Phys. Soc. Jpn 25 (1968) 1679.

[24] BATSON P.E., S. S. Commun. 37 (1980) 477.

[25] BATSON P.E., Surf. Sci. 156 (1985) 720.

[26 KAISER W.J., LOGOTHETIS E.M. and Wegener L.E., J. Phys. C 18 (1985) L837.

[27] Kaiser W.J., Logothetis E.M. and Wegener L.E., Sol. St. Commun. 57 (1986) 7. 